\title{
Heart Rate Variability and Metabolic Syndrome in Hospitalized Patients with Schizophrenia
}

\author{
Lee, Kyunghee ${ }^{1} \cdot$ Park, Jeongeon ${ }^{2} \cdot$ Choi, Jeongim ${ }^{3} \cdot$ Park, Chang Gi ${ }^{4}$ \\ ${ }^{1}$ Professor, College of Nursing, Keimyung University, Daegu \\ ${ }^{2}$ Full-time Lecturer, Department of Nursing, Gyeongsan University College, Gyeongsan \\ ${ }^{3}$ Staff Nurse, Keimyung University Dongsan Medical Center, Daegu, Korea \\ ${ }^{4}$ Statistian, College of Nursing, University of Illinois at Chicago, USA
}

Purpose: Reduced heart rate variability significantly increases cardiovascular mortality. Metabolic syndrome increases the cardiac autonomic dysfunction. Recently, increasing cardiovascular mortality has been reported in patients with schizophrenia. This study was done to compare heart rate variability between adults with and without schizophrenia and to compare the relationship of heart rate variability to metabolic syndrome in hospitalized patients with schizophrenia. Methods: This was a descriptive and correlational study in which 719 adults without schizophrenia and 308 adults with schizophrenia took part between May and June 2008. We measured the following: five-minute heart rate variability; high-frequency, low-frequency, the ratio of low-frequency to high-frequency, and the Standard Deviation of all the normal RR intervals. Data was also collected on metabolic syndrome, abdominal obesity, triglycerides, HDL cholesterol, blood pressure and fasting glucose. Results: The Standard Deviation of all the normal RR intervals values of heart rate variability indices were $1.53 \pm 0.18$. The low-frequency and high-frequency values of heart rate variability indices were significantly higher in hospitalized patients with schizophrenia $(3.89 \pm 1.36 ; 3.80 \pm 1.20)$ than those in the healthy participants $(2.20 \pm 0.46 ; 2.10 \pm 0.46)$. There were no significant differences between the schizophrenic patients with and without metabolic syndrome. Conclusion: The results of this study indicate that schizophrenia patients have significantly lower cardiac autonomic control, but they have significantly higher low-frequency and high-frequency values than those of healthy adults. Use of antipsychotic drug may affect the autonomic nervous system in schizophrenic patients. Metabolic syndrome was not associated with cardiac autonomic control in schizophrenia patients.

Key words: Metabolic syndrome X, Schizophrenia

\section{INTRODUCTION}

Heart rate variability (HRV) represents cardiac autonomic control and reduced HRV significantly increases cardiovascular mortality (La Rovere, Bigger, Marcus, Mortara, \& Schwartz, 1998; La Rovere et al., 2001). It is also generally known that time and frequency domain indices quantifying HRV are reduced in conditions such as diabetes (Kudat et al., 2006) and obesity (Karason, Molgaard, Wikstrand, \& Sjostrom, 1999; Kim et al., 2005). HRV provides quantitative infor- mation about the autonomic function and balance of the autonomic nervous system (ANS) through non-invasive indices of cardiac autonomic modulation (van Ravenswaaij-Arts, Kollee, Hopman, Stoelinga, \& van Geijn, 1993). A majority of studies have focused on patients with the diagnoses of coronary disease and neuropathies, but in recent years investigation of cardiac autonomic function has extended into other conditions including inpatients with schizophrenia in whom increased cardiovascular mortality was reported (Kelly et al., 2010).

\footnotetext{
*This study was funded by Keimyung Bisa research fund in 2007.

Address reprint requests to: Lee, Kyunghee

College of Nursing, Keimyung University, 1095 Dalgubeoldae-ro, Dalseo-gu, Daegu 704-701, Korea

Tel: +82-53-580-3927 Fax:+82-53-580-3916 E-mail:khl645@kmu.ac.kr

Received: May 31, 2011 Revised: June 20, 2011 Accepted: December 19, 2011
} 
Representative values of HRV indices include Standard Deviation of all normal RR intervals (SDNN), low-frequency (LF) band (0.04$0.15 \mathrm{~Hz}$ in humans) and the high-frequency (HF) band (0.16-0.4 Hz in humans) (Task Force of the European Society of Cardiology and the North American Society of Pacing and Electrophysiology, 1996). The SDNN is a strong indicator of the cardiovascular stability and ability of the ANS to control the body system (Montano et al., 1994). The representation of LF and HF in normalized units emphasizes the controlled and balanced behavior of the two branches of the autonomic nervous system. The ratio of low to high frequency $(\mathrm{LF} / \mathrm{HF})$ is the ratio of sympathetic nervous system (SNS) to parasympathetic nervous system (PNS) (Task Force of the European Society of Cardiology and the North American Society of Pacing and Electrophysiology, 1996).

The relationship between HRV and metabolic syndrome in general population was elucidated by many researchers (Kuch et al., 2001; Lee et al., 2004; Oh, Hong, Sung, \& Barrett-Connor, 2004).

HRVs in diabetes and obesity were found to be associated with metabolic syndrome and it was found to increase the cardiac autonomic dysfunction (Astrup et al., 2006). The use of antipsychotics in schizophrenia apparently to improve psychotic symptoms, the metabolic effects of atypical antipsychotics have received particular attention in the literature as accrued experience demonstrates marked differences between various agents in their risk for weight gain and changes in serum triglycerides and glycemic control (Meyer, 2001). A high prevalence of metabolic syndrome has been reported among schizophrenic patients (McEvoy et al., 2005; Meyer et al., 2005; Sugawara et al., 2011). In the study of Sugawara et al., they compared the metabolic syndrome prevalence between inpatients and outpatients among schizophrenic population in Japan. The result was reported that outpatients with schizophrenia or schizoaffective disorder in Japan had a high prevalence of metabolic syndrome compared to inpatients.

However, limited studies exist in the literature that examined the relationship between HRV and metabolic syndrome in schizophrenic patients. This study aimed to fill the gap in the literature. Specific aims of the study were to compare heart rate variability between healthy adults and hospitalized patients with schizophrenia, and to examine the relationships of HRV to metabolic syndrome in hospitalized patients with schizophrenia.

\section{METHODS}

\section{Study population}

Data were collected from 719 healthy adults (H participants: 510 male patients and 209 female patients) who visited the outpatient clinic of a major university hospital for annual checkup and 308 patients (207 male patients and 100 female patients) who were hospitalized with the diagnosis of DSM-IV schizophrenia (S participants) at two hospitals and one sanatorium in two cities that were geographically closely.

\section{Data collection procedure}

Data were collected from H participants first (March 1 to June 30 in 2008), and then S participants (May 1 to June 30 in 2008). Research assistants who were trained for the study collected: Body Mass Index (BMI), triglycerides, High-Density Lipoprotein (HDL) cholesterol, blood pressure and fasting glucose. They then measured the following as the indices of HRV: five-minute beat-to-beat heart rate which were, collected while they were sitting; resting HF (0.16-0.4 Hz) and LF (0.04-0.15 Hz) spectral powers; the ratio of LF to HF, and the SDNN. The same elements were measured in healthy participants as they were parts of their routine check-ups.

Metabolic syndrome was measured in hospitalized patients with schizophrenia and used the classification that used appropriate cutoffs for overweight in Asian populations. Metabolic syndrome was defined as having at least 3 of the following parameters after 3 months of therapeutic lifestyle changes (Grundy et al., 2004)

1) Abdominal obesity (waist circumference male $\geq 90 \mathrm{~cm}$, female $\geq 80 \mathrm{~cm}$ ) (WHO, IASO, \& IOTF, 2000)

2) Triglycerides $\geq 150 \mathrm{mg} / \mathrm{dL}$

3) HDL cholesterol $\leq 40 \mathrm{mg} / \mathrm{dL}$ (men) and $\leq 50 \mathrm{mg} / \mathrm{dL}$ (women)

4) Blood pressure: $\geq 130 / \geq 85 \mathrm{mmHg}$

5) Fasting glucose: $\geq 110 \mathrm{mg} / \mathrm{dL}$

Sociodemographic data were obtained from their medical records. The participants were asked not to eat, drink alcoholics or caffeinated beverages and smoke for at least 12 hours before the data collection. The data collection and HRV analysis were recorded by SA-2000E (Medicore Co., Korea). BMI was examined by BMI Measuring System (Fanics), and the blood pressure was checked by BP-203RV II 
(Colin). The fasting blood sugar was measured by Toshiba (Japan). Sitting blood pressure was measured by the random zero sphygnomanometer after a 5-minute rest.

Permission for accessing and using the data was obtained from the directors of the hospitals and outpatient clinic (they didn't have formalized Institutional Review Board [IRB] procedure). All H and S participants who were asked to participate in the research agreed and signed the informed consent. Consents from $S$ participants were obtained from family members over the telephone.

ANS dysfunction and reduced HRV have been reported in a wide variety of normal people, but have not been well characterized in schizophrenia. We recorded cardiac activity and assessed HRV in hospitalized schizophrenia (SCZ) patients compared to healthy comparison $(\mathrm{HC})$ participants.

\section{Non-spectral HRV analysis}

In the time domain analysis of HRV, the standard deviation of all RR intervals (SDNN), the square root of the mean squared differences of successive normal sinus intervals (RMSSD) were computed. SDNN estimates global HRV, mediated by sympathetic and parasympathetic nervous systems.

\section{Spectral HRV analysis}

In the frequency domain, spectral analysis was performed using fast Fourier transform (Task Force of the European Society of Cardiology and the North American Society of Pacing and Electrophysiology, 1996) and included low-frequency spectral powers (LF, 0.04-0.15 $\mathrm{Hz})$ resting high-frequency spectral powers $(\mathrm{HF}, 0.16-0.4 \mathrm{~Hz})$ and the $\mathrm{LF} / \mathrm{HF}$ ratio $(\mathrm{L} / \mathrm{H})$. Mathematically, the process of Fourier analysis is represented by the Fast Fourier Transform, which is the sum over all time of the signal $f(t)$ multiplied by a complex exponential. The results of the transform are the Fourier coefficients; the squared coefficients for each frequency are the power spectral density of the signal $\mathrm{f}(\mathrm{t})$ (Task Force of the European Society of Cardiology and the North American Society of Pacing and Electrophysiology, 1996).

\section{Statistical analysis}

For statistical analyses, SPSS for Windows Program (version 15.0) and Stata ${ }^{\circledR} 11.1$ were used. All data were tested for normality using the Kolmogorov-Smimov test. To normalize skewed HRV variables, a logarithmic transform was applied. Group differences of general characteristics between $\mathrm{H}$ and S participants were analyzed by Chisquare test.

Logistic regression analyses were used for the values of adjusted odds ratio (AOR) and 95\% confidence interval (CI) by using median of HRV variables between $H$ and $S$ participants.

Kernel Density Estimation was used for comparisons of the distributions of LF and HF between the two groups ( $\mathrm{H}$ and $\mathrm{S}$ participants group). Statistical significance was determined as $p<.05$.

\section{RESULTS}

\section{Sample Characteristics}

The determinants of short-period HRV in $719 \mathrm{H}$ participants and $307 \mathrm{~S}$ participants are shown in Table 1 . The mean age of $\mathrm{H}$ participants was $49.5 \pm 8.7$ years, and $S$ participants was $51.1 \pm 9.8$ years, and the age difference was significant between the two groups $(p<.001)$ (Table 1).

\section{Linear HRV indices between $H$ participants and $S$ participants}

When age was used as covariate, $\mathrm{H}$ participants showed significantly higher SDNN and RMSSD values than those of S participants $(p<.001)$, while S participants showed significantly higher LF and HF values $(p<.001)$ than those of $H$ participants. SDs were similar for SDNN and RMSSD in both groups, but mean SD values of both LF and HF of S participants were nearly 3 times larger than those of

Table 1. Characteristics of Study Population $(N=1,026)$

\begin{tabular}{|c|c|c|c|c|c|c|}
\hline & & $\begin{array}{l}\text { Healthy } \\
\text { participants } \\
(n=719)\end{array}$ & $\begin{array}{l}\text { Inpatients with } \\
\text { schizophrenia } \\
(n=307)\end{array}$ & $\begin{array}{c}\text { Total } \\
(\mathrm{n}=1,026)\end{array}$ & $x^{2}$ & $p$ \\
\hline & & $\mathrm{n}(\%)$ & n (\%) & n (\%) & & \\
\hline Age & $<41$ & 97 (13.5) & $33(10.7)$ & $130(12.7)$ & 18.65 & $<.001$ \\
\hline \multirow{3}{*}{$(y r)$} & $41-50$ & $289(40.2)$ & $113(36.8)$ & 402 (39.2) & & \\
\hline & $51-60$ & $279(38.8)$ & $111(36.2)$ & $390(38.0)$ & & \\
\hline & $60>$ & $54(7.5)$ & 50 (16.3) & $104(10.1)$ & & \\
\hline \multirow{2}{*}{ Gender } & Males & $510(70.9)$ & $207(67.4)$ & 717 (69.9) & 1.26 & .262 \\
\hline & Females & 209 (29.1) & 100 (32.6) & 309 (30.1) & & \\
\hline
\end{tabular}


H participants. However, there were no significant differences in the ratio of $\mathrm{LF} / \mathrm{HF}$ values between two groups $(p>.05)$. These are shown in Table 2 .

In logistic regression analyses, $\mathrm{S}$ participants were significantly associated with RMSSD (AOR, 0.52; 95\% CI, 0.35-0.78) and SDNN (AOR, 2.00; 95\% CI, 1.37-2.90). But they were not significantly associated with LF (AOR, 1.11; 95\% CI, 0.75-1.63), HF (AOR, 1.10; 95\% CI, 0.73-1.65) and LF/HF (AOR, 0.85; 95\% CI, 0.62-.1.18).

Figures 1 and 2 show that the mean and standard deviation values of linear LF in H participants were $2.20 \pm 0.46$ while those of S participants were $3.89 \pm 1.36$; the mean and standard deviation values of linear HF in $\mathrm{H}$ participants were $2.10 \pm 0.46$ while those in S participants were $3.80 \pm 1.20$. As shown above, the distribution patterns of LF and HF between two groups were similar, although distribution patterns of LF and HF in S participants showed broader range than those of $\mathrm{H}$ participants. This indicates that $\mathrm{S}$ participants had significantly higher LF and HF values than those of H participants.

Table 2. Values of Linear Heart Rate Variability Indices between $\mathrm{H}$ Participants and S Participants $(N=1,026)$

\begin{tabular}{|c|c|c|c|c|c|}
\hline \multirow[t]{2}{*}{ Variable } & $\begin{array}{l}\text { Healthy } \\
\text { participants } \\
(\mathrm{n}=719)\end{array}$ & $\begin{array}{l}\text { Inpatients with } \\
\text { schizophrenia } \\
(n=307)\end{array}$ & \multirow[t]{2}{*}{$F^{*}$} & \multirow[t]{2}{*}{$p$} & \multirow[t]{2}{*}{$\mathrm{AOR}(95 \% \mathrm{Cl})^{\dagger}$} \\
\hline & Mean (SD) & Mean (SD) & & & \\
\hline SDNN & $1.53(0.18)$ & $1.29(0.23)$ & 329.81 & $<.001$ & $2.00(1.37-2.90)$ \\
\hline RMSSD & $1.38(0.21)$ & $1.18(0.24)$ & 163.17 & $<.001$ & $0.52(0.35-0.78)$ \\
\hline LF & $2.20(0.46)$ & $3.89(1.36)$ & 910.03 & $<.001$ & $1.11(0.75-1.63)$ \\
\hline $\mathrm{HF}$ & $2.10(0.46)$ & $3.80(1.20)$ & 1123.63 & $<.001$ & $1.10(0.73-1.65)$ \\
\hline LF/HF & $1.08(0.27)$ & $1.06(0.36)$ & 0.94 & .333 & $0.85(0.62-1.18)$ \\
\hline
\end{tabular}

${ }^{*}$ Covariate: Age; 'Logistic regression analyses adjusted with all above variables.

AOR=Adjusted odds ratio; $95 \% \mathrm{Cl}=95 \%$ confidence interval; SDNN = Standard deviation of all normal RR intervals; RMSSD=Root mean square of successive differences; LF=Low-frequency; HF=High-frequency; LF/HF: The ratio of low to high frequency.

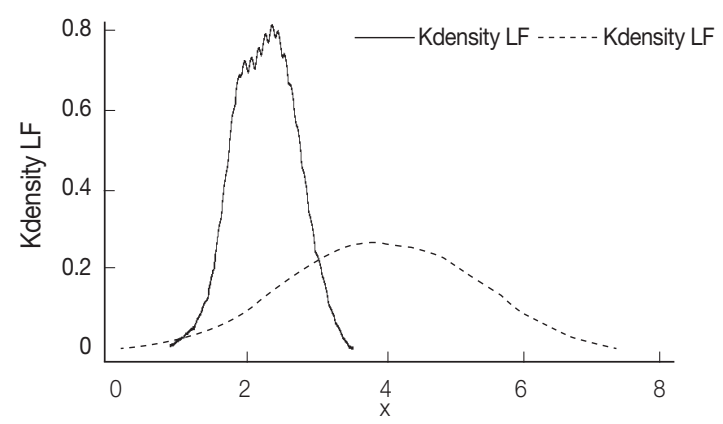

Figure 1. Kernel density estimation of LF between two groups. Left: Healthy participants; Right: Inpatients with schizophrenia.

\section{Differences in linear HRV indices according to metabolic syndrome between healthy participants and inpatients with schizophrenia groups}

Table 3 shows the differences in HRV indices according to metabolic syndrome between the two groups. SDNN, RMSSD, LF and HF values in the healthy subject group with metabolic syndrome (H-1 group) were significantly lower than those in the healthy subject group without metabolic syndrome (H-2 group). But there were no significant differences between the inpatient group with metabolic syndrome (S-1 group) and the inpatient group without metabolic syndrome (S-2 group).

In logistic regression analyses, all participants with or without metabolic syndrome were significantly associated with SDNN (AOR, 1.70; 95\% CI, 1.09-2.64). Also, H participants group were significantly associated with SDNN (AOR, 2.13; 95\% CI, 1.24-3.66) according to metabolic syndrome. But, in S participants group, there were not significantly associated with RMSSD (AOR, 0.65; 95\% CI, 0.27-1.57), HF (AOR, 1.31; 95\% CI, 0.53-3.23), SDNN (AOR, 1.21; 95\% CI, 0.49-3.01) according to metabolic syndrome.

\section{DISCUSSION}

The objective of the current study used HRV to test autonomic function of the heart, and compared HRV between healthy adults and hospitalized patients with schizophrenia in Korea.

Hospitalized patients with schizophrenia exhibited a decrease of SDNN and RMSSD in HRV compared to healthy participants group. As expected, SDNN and RMSSD of healthy participants group showed significantly higher values than those of inpatients with

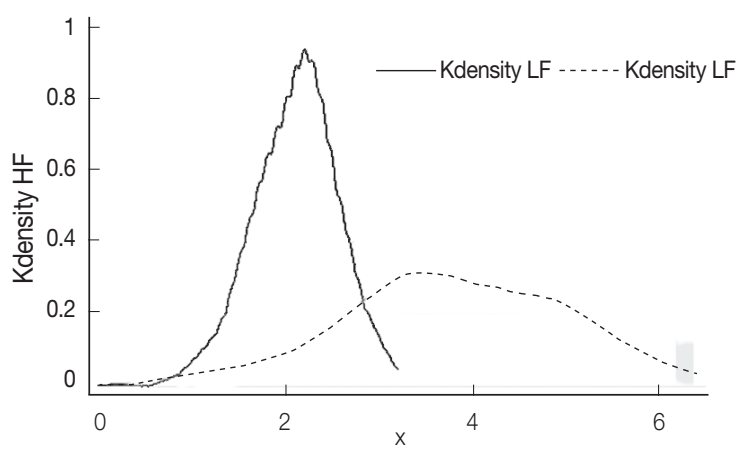

Figure 2. Kernel density estimation of HF between two groups. Left: Healthy participants; Right: Inpatients with schizophrenia. 
Table 3. Differences in Heart Rate Variability Indices according to the Metabolic Factor between Healthy Participants and Inpatients with Schizophrenia Groups

$(N=1,026)$

\begin{tabular}{|c|c|c|c|c|c|c|c|c|}
\hline & \multicolumn{4}{|c|}{ Healthy participants } & \multicolumn{4}{|c|}{ Inpatients with schizophrenia } \\
\hline & $\mathrm{H}-1$ & $\mathrm{H}-2$ & \multirow{2}{*}{$F^{*}(p)$} & \multirow{2}{*}{$\mathrm{AOR}^{\dagger}(95 \% \mathrm{Cl})$} & S-1 & S-2 & \multirow{2}{*}{$F^{*}(p)$} & \multirow{2}{*}{$\mathrm{AOR}^{\dagger}(95 \% \mathrm{Cl})$} \\
\hline & Mean (SD) & Mean (SD) & & & Mean (SD) & Mean (SD) & & \\
\hline SDNN & $1.46(0.18)$ & $1.55(0.18)$ & $19.33(<.001)$ & $2.13(1.24-3.66)$ & $1.27(0.23)$ & $1.29(0.23)$ & $0.86(.354)$ & $1.21(0.49-3.01)$ \\
\hline RMSSD & $1.33(0.25)$ & $1.39(0.20)$ & $6.28(<.05)$ & $0.88(0.51-1.53)$ & $1.18(0.27)$ & $1.19(0.24)$ & $0.06(.802)$ & $0.65(0.27-1.57)$ \\
\hline LF & $2.07(0.48)$ & $2.23(0.45)$ & $6.86(<.05)$ & $1.33(0.75-2.38)$ & $3.78(1.35)$ & $3.92(1.37)$ & $0.01(.916)$ & $0.99(0.41-2.34)$ \\
\hline HF & $1.98(0.51)$ & $2.13(0.44)$ & $8.12(<.05)$ & $0.97(0.54-1.72)$ & $3.80(1.30)$ & $3.80(1.18)$ & $0.69(.408)$ & $1.31(0.53-3.23)$ \\
\hline LF/HF & $1.09(0.32)$ & $1.08(0.27)$ & $0.24(.329)$ & $0.85(0.52-1.40)$ & $1.04(0.33)$ & $1.07(0.36)$ & $0.38(.539)$ & $1.05(0.54-2.08)$ \\
\hline
\end{tabular}

${ }^{*}$ Covariate: Age; ${ }^{\dagger}$ Logistic regression analyses adjusted with all above variables.

$\mathrm{AOR}=$ Adjusted odds ratio; $95 \% \mathrm{Cl}=95 \%$ confidence interval; SDNN=Standard deviation of all normal RR intervals; RMSSD=Root mean square of successive

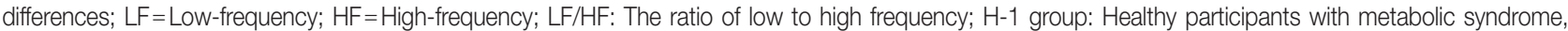
$\mathrm{n}=115$; H-2 group: Healthy participants without metabolic syndrome, $n=604$; S-1 group: Inpatients with schizophrenia with metabolic syndrome, $n=61 ; \mathrm{S}-2$ group: Inpatients with schizophrenia without metabolic syndrome, $n=246$.

schizophrenia. This suggests that healthy participants showed more cardiovascular stability and ability of the ANS to control the body system than the hospitalized patients with schizophrenia. Schizophrenia is a mental disorder with typical characteristics of behaviorally symptoms (such as psychomotor retardation, lack of insight, poor attention and impulse control) as positive symptoms (including hallucinations, delusions and thought disorder), negative symptoms (such as social withdrawal, apathy and emotional blunting) and other psychopathological symptom. It is a severe mental disorder that requires lifelong treatment, and therefore information on the cardiovascular safety and tolerance of antipsychotics is of significant clinical importance. A growing number of reports have observed HRV dysfunction in schizophrenia (Bar et al., 2005; Birkhofer, Alger, Schmid, \& Forstl, 2007; Boettger et al., 2006). In schizophrenia, autonomic dysregulation is associated with more severe psychiatric symptoms, suggesting HRV dysfunction in this disorder may be dependent on the phase of the illness (Bar et al.; Boettger et al.; Henry, Minassian, Paulus, Geyer, \& Perry, 2010).

Decreased HRV was found to be associated with a pathological conditions like metabolic syndrome (Liao, Carnethon, Evans, Cascio, \& Heiss, 2002) and low SDNN was reported as having an independent prognostic value for cardiac mortality (La Rovere et al., 2001). In logistic regression analyses, total participants without metabolic syndrome were significantly associated with SDNN (AOR, 1.70; 95\% CI, 1.09-2.64). Taken as a whole, these data indicate that SDNN of total participants group without metabolic syndrome demonstrated significantly higher values than those of total participants group with metabolic syndrome. This suggests that total participants group without metabolic syndrome showed more cardiovascular stability and ability of the ANS to control the body system than total participants group with metabolic syndrome. As expected, H participants group were significantly associated with SDNN (AOR, 2.13; 95\% CI, 1.24-3.66) according to metabolic syndrome. But, these results differ from the reports describing HRV in S participants group. S participants group were not significantly associated with RMSSD (AOR, 0.65; 95\% CI, 0.27-1.57), HF (AOR, 1.31; 95\% CI, 0.53-3.23), SDNN (AOR, 1.21; 95\% CI, 0.49-3.01) according to metabolic syndrome. These results suggest that $S$ participants group are not significantly associated with the values of HRV indices according to metabolic syndrome. This may be explained, in part, by the fact that all S participants group in this study were on various anti-psychotic medications with different dosages. Although $S$ participants group exhibited decreased SDNN compared to healthy comparison participants, they showed an increase in the linear HF and LF indices in contradiction to the current findings.

The distributions of the linear HF and LF indices between the two groups were compared by the Kernel Density Estimation. Hospitalized patients with schizophrenia showed significantly higher LF and HF values than that of healthy participants. Using the criteria of the Adult Treatment Panel III of the National Cholesterol Education Program (Grundy et al., 2004), the prevalence rate of metabolic syndrome in hospitalized patients with schizophrenia was 19.9\%, in same inpatient population were $36-37 \%$, which was higher than that of general populations (Lee et al., 2004; Suvisaari et al., 2007). In Korea, the prevalence of metabolic syndrome was $16.0 \%$ in men and $10.7 \%$ in women aged $30-80$ years (Oh, Hong, Sung, \& Barrett-Con- 
nor, 2004).

No significant differences were noted in HRV between S participants with and without metabolic syndrome suggesting that the metabolic syndrome in S participants is not associated with decreased HRV. Their relationship didn't show the significance, but show the tendency of association with decreased HRV.

These finding has implications with the fact that significantly higher LF and HF indices shown in S participants than that of healthy participants in this study may be explained in part by the use of antipsychotic drugs that all S participants were taking. These medicines may have affected autonomic nervous system as a mediating factor between metabolic syndrome and HRV in these patients. Agelink et al. (2001) suggested neurocardiac effects of atypical neuroleptics (amisulpride, olanzapine, clozapine and sertindole) on ANF should exert distinct effects on ANF depending on the extent to which anticholinergic and/or antiadrenergic effects. In this study, S participants were on various antipsychotic drugs. Heart rate deceleration capacity is significantly reduced in schizophrenic patients treated with antipsychotics and may serve as an indicator of increased cardiovascular mortality risk (Birkhofer, Alger, Schmid, \& Forstl, 2007).

Patients treated with neuroleptic medications, especially clozapine, showed autonomic dysregulation and cardiac repolarization changes. Physicians should be aware of this adverse reaction (Cohen, Loewenthal, Matar, \& Kotler, 2001).

Other result showed significant increases in the mean, variance and HF of RR intervals in the amisulpride group, but not in the olanzapine group (Wang, Yang, Bai, \& Kuo, 2008). These results indicate that amisulpride has a more vagotonic effect, suggesting greater cardiovascular safety as compared with olanzapine when participants are switched from typical antipsychotic agents. In the present study, HRV was investigated during treatment with olanzapine. After 4 weeks of olanzapine medication, HRV was assessed during different sleep stages both in the time and frequency domains. Only slight changes in HRV were shown during treatment, and appeared to be independent of sleep stages. Spectral analysis indicated a slight shift of the sympathovagal balance in favour of the sympathetic tone, which was consistent with an elevation of heart rate in the time domain; total HRV was not altered. These changes are in accordance with olanzapine's receptor profile exerting anticholinergic and anti-adrenergic properties (Mann et al., 2004).

These findings suggest that the effects of combination psychotro- pic drug treatment on HRV are mainly due to their anticholinergic properties (Ikawa et al., 2001). We cannot rule out potential effects of antipsychotic drugs or schizophrenic symptoms because the metabolic syndrome may be associated with HRV differently in inpatients with schizophrenia due to antipsychotic polypharmacy (Agelink et al., 2001).

Major limitations of this study include: lack of information on medication profile of hospitalized patients with schizophrenia; the use of medical records that might have included incomplete information; and the use of convenience samples in three hospital settings in two different cities although they were nearby to each other with similar sociodemographic profiles.

\section{CONCLUSIONS}

Hospitalized patients with schizophrenia showed significantly lower cardiac autonomic control, but they had significantly higher LF and HF values than those of healthy participants. A reduced cardiac autonomic control was not associated with metabolic syndrome in hospitalized patients with schizophrenia. Antipsychotic polypharmacy may have affected autonomic nervous system function as a mediating factor between metabolic syndrome and HRV in these patients.

\section{REFERENCES}

Agelink, M. W., Majewski, T., Wurthmann, C., Lukas, K., Ullrich, H., Linka, T., et al. (2001). Effects of newer atypical antipsychotics on autonomic neurocardiac function: A comparison between amisulpride, olanzapine, sertindole, and clozapine. Journal of Clinical Psychopharmacology, 21, 8-13.

Astrup, A. S., Tarnow, L., Rossing, P., Hansen, B. V., Hilsted, J., \& Parving, H. H. (2006). Cardiac autonomic neuropathy predicts cardiovascular morbidity and mortality in type 1 diabetic patients with diabetic nephropathy. Diabetes Care, 29, 334-339. doi: 10.2337/diacare.29.02.06. dc05-1242

Bar, K. J., Letzsch, A., Jochum, T., Wagner, G., Greiner, W., \& Sauer, H. (2005). Loss of efferent vagal activity in acute schizophrenia. Journal of Psychiatric Research, 39, 519-527.

Birkhofer, A., Alger, P., Schmid, G., \& Forstl, H. (2007). The cardiovascular risk of schizophrenic patients. Neuropsychiatry, 21, 261-266.

Boettger, S., Hoyer, D., Falkenhahn, K., Kaatz, M., Yeragani, V. K., \& Bar, K. J. (2006). Altered diurnal autonomic variation and reduced vagal information flow in acute schizophrenia. Clinical Neurophysiology, 117, 2715-2722. doi: 10.1016/j.clinph.2006.08.009

Cohen, H., Loewenthal, U., Matar, M., \& Kotler, M. (2001). Association of 
autonomic dysfunction and clozapine. Heart rate variability and risk for sudden death in patients with schizophrenia on long-term psychotropic medication. The British Journal of Psychiatry, 179, 167-171.

Grundy, S. M., Cleeman, J. I., Merz, C. N., Brewer, H. B. Jr., Clark, L. T., Hunninghake, D. B., et al. (2004). Implications of recent clinical trials for the National Cholesterol Education Program Adult Treatment Panel III guidelines. Circulation, 110, 227-239.

Henry, B. L., Minassian, A., Paulus, M. P., Geyer, M. A., \& Perry, W. (2010). Heart rate variability in bipolar mania and schizophrenia. Journal of Psychiatric Research, 44, 168-176. doi: 10.1016/j.jpsychires.2009.07.011

Ikawa, M., Tabuse, H., Ueno, S., Urano, T., Sekiya, M., \& Murakami, T. (2001). Effects of combination psychotropic drug treatment on heart rate variability in psychiatric patients. Psychiatry and Clinical Neurosciences, 55, 341-345. doi: 10.1046/j.1440-1819.2001.00873.x

Karason, K., Molgaard, H., Wikstrand, J., \& Sjostrom, L. (1999). Heart rate variability in obesity and the effect of weight loss. American Journal of Cardiology, 83, 1242-1247. doi: 10.1016/S0002-9149(99)00066-1

Kelly, D. L., McMahon, R. P., Liu, F., Love, R. C., Wehring, H. J., Shim, J. C., et al. (2010). Cardiovascular disease mortality in patients with chronic schizophrenia treated with clozapine: A retrospective cohort study. Journal of Clinical Psychiatry, 71, 304-311. doi: 10.4088/JCP.08m04718yel

Kim, J. A., Park, Y. G., Cho, K. H., Hong, M. H., Han, H. C., Choi, Y. S., et al. (2005). Heart rate variability and obesity indices: Emphasis on the response to noise and standing. Journal of the American Board of Family Practice, 18, 97-103. doi: 10.1152/japplphysiol.01275.2005

Kuch, B., Hense, H. W., Sinnreich, R., Kark, J. D., von Eckardstein, A., Sapoznikov, D., et al. (2001). Determinants of short-period heart rate variability in the general population. Cardiology, 95, 131-138.

Kudat, H., Akkaya, V., Sozen, A. B., Salman, S., Demirel, S., Ozcan, M., et al. (2006). Heart rate variability in diabetes patients. Journal of International Medical Research, 34, 291-296.

La Rovere, M. T., Bigger, J. T. Jr., Marcus, F. I., Mortara, A., \& Schwartz, P. J. (1998). Baroreflex sensitivity and heart-rate variability in prediction of total cardiac mortality after myocardial infarction. ATRAMI (Autonomic Tone and Reflexes After Myocardial Infarction) Investigators. Lancet, 351, 478-484. doi: 10.1016/S0140-6736(97)11144-8

La Rovere, M. T., Pinna, G. D., Hohnloser, S. H., Marcus, F. I., Mortara, A., Nohara, R., et al. (2001). Baroreflex sensitivity and heart rate variability in the identification of patients at risk for life-threatening arrhythmias: Implications for clinical trials. Circulation, 103, 2072-2077.

Lee, W. Y., Park, J. S., Noh, S. Y., Rhee, E. J., Kim, S. W., \& Zimmet, P. Z. (2004). Prevalence of the metabolic syndrome among 40,698 Korean metropolitan subjects. Diabetes Research and Clinical Practice, 65, 143-149.

Liao, D., Carnethon, M., Evans, G. W., Cascio, W. E., \& Heiss, G. (2002). Lower heart rate variability is associated with the development of coronary heart disease in individuals with diabetes: The atherosclerosis risk in communities (ARIC) study. Diabetes, 51, 3524-3531.
Mann, K., Rossbach, W., Muller, M. J., Muller-Siecheneder, F., Ru, H., \& Dittmann, R. W. (2004). Heart rate variability during sleep in patients with schizophrenia treated with olanzapine. International Clinical Psychopharmacology, 19, 325-330.

McEvoy, J. P., Meyer, J. M., Goff, D. C., Nasrallah, H. A., Davis, S. M., Sullivan, L., et al. (2005). Prevalence of the metabolic syndrome in patients with schizophrenia: Baseline results from the Clinical Antipsychotic Trials of Intervention Effectiveness (CATIE) schizophrenia trial and comparison with national estimates from NHANES III. Schizophrenia Research, 80, 19-32. doi: 10.1016/j.schres.2005.07.014

Meyer, J. M. (2001). Effects of atypical antipsychotics on weight and serum lipid levels. Journal of Clinical Psychiatry, 62, 27-34.

Meyer, J. M., Nasrallah, H. A., McEvoy, J. P., Goff, D. C., Davis, S. M., Chakos, M., et al. (2005). The Clinical Antipsychotic trials Of Intervention Effectiveness (CATIE) Schizophrenia trial: Clinical comparison of subgroups with and without the metabolic syndrome. Schizophrenia Research, 80, 9-18. doi: 10.1016/j.schres.2005.07.015

Montano, N., Gneddhi, R.T., Porta, A., Lombardi, F., Pagani, M., \& Malliani, A. (1994). Power spectrum analysis of heart rate variability to assess the changes in sympathovagal balance during graded orthostatic tilt. Circulation, 90, 1826-1831.

Oh, J. Y., Hong, Y. S., Sung, Y. A., \& Barrett-Connor, E. (2004). Prevalence and factor analysis of metabolic syndrome in an urban Korean population. Diabetes Care, 27, 2027-2032. doi: 10.2337/diacare.27.8.2027

Sugawara, N., Yasui-Furukori, N., Sato, Y., Kishida, I., Yamashita, H., Saito, M., et al. (2011). Comparison of prevalence of metabolic syndrome in hospital and community-based Japanese patients with schizophrenia. Annals of General Psychiatry, 10(21), 1-8. doi: 10.1186/1744-859X-1021

Suvisaari, J. M., Saarni, S. I., Perala, J., Suvisaari, J. V., Harkanen, T., Lonnqvist, et al. (2007). Metabolic syndrome among persons with schizophrenia and other psychotic disorders in a general population survey. Journal of Clinical Psychiatry, 68, 1045-1055.

Task Force of the European Society of Cardiology and the North American Society of Pacing and Electrophysiology. (1996). Heart rate variability: Standards of measurement, physiological interpretation, and clinical use. Circulation, 93, 1129-1136.

Van Ravenswaaij-Arts, C. M., Kollee, L. A., Hopman, J. C., Stoelinga, G. B., \& van Geijn, H. P. (1993). Heart rate variability. Annals of Internal Medicine, 118, 436-447.

WHO, IASO, \& IOTF. (2000). The Asia-Pacific perspective: Redefining obesity and its treatment. Australia: Health Communications, 1-56.

Wang, Y. C., Yang, C. C., Bai, Y. M., \& Kuo, T. B. (2008). Heart rate variability in schizophrenic patients switched from typical antipsychotic agents to amisulpride and olanzapine: 3-month follow-up. Neuropsychobiology, 57, 200-205. doi: 10.1159/000149818 